\section{Keratinozyten - Opfer und Täter zugleich}

\section{Keratinozyten sind nicht nur wehrloses Opfer von T-Zellen. Sie scheinen ihrerseits Ekzemreaktionen zu beeinflussen, indem sie die Proliferation und Zytokinfreisetzung von T-Zellen modulieren.}

1 eute ist bekannt, dass im Rahmen allergischer Kontaktekzeme wie auch beim atopischen Ekzem T-Zellen in die Haut infiltrieren und dort die Apoptose von Keratinozyten auslösen. Stefanie Förster, München, ging der Frage nach, ob Keratinozyten möglicherweise in der Lage sind, die T-Zell-Aktivität zu modulieren. Dazu kam ein autologes In-vitroModell der Ekzemreaktion zum Einsatz: Nickel- bzw. Phl-p-1-spezifische T-ZellKlone wurden von Nickel- bzw. Phleumpratense-sensiblisierten Probanden generiert und in einer Ko-Kultur mit autologen Antigen-präsentierenden Zellen und dem jeweiligen Antigen inkubiert. Als Effektorparameter der T-Zell-Klone wurden spezifische Proliferation und Zytokinproduktion gemessen. Die modulatorische Wirkung von Keratinozyten auf diese Parameter wurde durch Zugabe

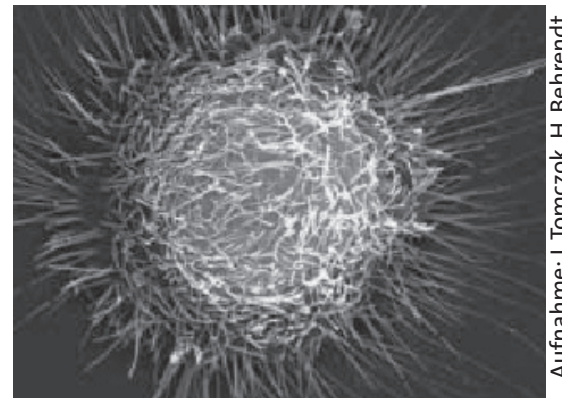

Humaner Keratinozyt (REM, Vergrößerung 2.000-fach)

autologer Keratinozyten zur Ko-Kultur und Vergleich mit dem Kontrollansatz ohne Keratinozyten bestimmt.

Im In-vitro-Modell der allergischen Kontaktdermatitis auf Nickel wurde die Proliferation der Nickel-spezifischen TZell-Klone signifikant durch die Anwe- senheit autologer Kerationzyten gehemmt. Es gab jedoch auch T-Zell-Klone, deren Proliferation verstärkt wurde, berichtete Förster. Die Freisetzung der Zytokine IL4 und IL-10 wurde einheitlich durch Keratinozyten gehemmt. IFN- $\gamma$ wurde nicht konstant reguliert.

Phl-p-1-spezifische T-Zell-Klone zeigten im In-vitro-Modell der atopischen Dermatitis auf Allergene ebenfalls eine Hemmung ihrer spezifischen Proliferation und der IL-4- und IL-10-Produktion, jedoch eine signifikante Induktion der IFN- $\boldsymbol{\gamma}$-Freisetzung in der Gegenwart autologer Keratinozyten. Dieser immunmodulatorische Effekt auf T-Zellen ist unabhängig vom Differenzierungsgrad der Keratinozyten und kann durch Fixierung der Keratinozyten mit Paraformaldehyd aufgehoben werden. Keratinozyten sezernieren somit lösliche Faktoren, die durch die Induktion der IFN- $\gamma$-Produktion in T-Zellen eine Amplifzierung und Chronifizierung der atopischen Ekzemläsion bedingen können.

abd

Förster S, Eyerich K, Ring J, Behrendt H, Traidl-Hoffmann C. Crosstalk of keratinocytes and T cells during eczematous skin reactions. 19. Mainzer Allergie-Workshop, Mainz, 16.-17. März 2007

\title{
Autoimmunprozesse bei atopischer Dermatitis?
}

\section{Bei Patienten mit atopischen Erkrankungen ist eine IgE-vermittelte Autoreaktivität wie auch eine Auslösbarkeit von Ekzemreaktionen durch humane Autoantigene beobachtet worden. Ein potentes Auto- antigen scheint die $\alpha$-Kette des Proteins NAC zu sein.}

\footnotetext{
$\mathrm{n}$ aktuellen Forschungsarbeiten konnten bei Patienten mit atopischer Dermatitis IgE-Autoantikörper gegen humane Antigene nachgewiesen werden. Die Autoreaktivität korrelierte dabei mit der Schwere der Erkrankung. Eines der Autoantigene, die dabei eine Rolle spielen könnten, ist Hom S2. Dabei handelt es sich um die $\alpha$-Kette des „human nascent polypeptide-associated complex" $(\alpha-$ NAC), ein intrazelluläres Protein, das als Transkriptions-Koaktivator agiert. Man
}

geht davon aus, dass das Kratzen aufgrund des Juckreizes bei der atopischen Dermatitis zu Hautschädigungen und dadurch zu einer Freisetzung von $\alpha$-NAC führt.

Eine Arbeitsgruppe aus Hannover screente nun Seren von 165 Patienten mit atopischer Dermatitis auf spezifische Antikörper gegen NAC. Gefunden wurde eine hohe Sensibilisierungsrate von $22 \%$, berichtete Dr. Annice Heratizadeh. Es konnte gezeigt werden, dass NAC zu einer Proliferation von Lymphozyten im peri- pheren Blut führt, und zwar sowohl bei Ekzempatienten wie auch bei gesunden Kontrollen. NAC induzierte - im Unterschied zur Mediumkontrolle - insbesondere die Proliferation der Haut-assoziierten $\mathrm{CLA}^{+}$- und CCR $4^{+}$-Lymphozyten.

Eine NAC-spezifische T-Zell-Reaktivität ließ sich auch in läsionaler Haut von Ekzempatienten nachweisen. Bbei dieser Lymphozytenproliferation handelte es sich um eine T-Zell-Rezeptor-vermittelte Immunantwort. Die T-Zell-Antwort auf NAC sei abhängig von vitalen Antigen-präsentierenden Zellen, erklärte Heratizadeh.

$a b d$

Heratizadeh A, Kienlin P, Mittermann I, Valenta R, Werfel T. Proliferation of skinhoming lymphocytes induced by the alpha-nascent-polypeptide-associated complex (NAC, Hom S2) in adult patients with atopic dermatitis. 19. Mainzer Allergie-Workshop, Mainz, 16.-17. März 2007 